\title{
Entropically Patchy Particles: Engineering Valence Through Shape Entropy
}

\author{
Greg van Anders, ${ }^{1}$ N. Khalid Ahmed, ${ }^{1}$ Ross Smith, ${ }^{2}$ Michael Engel, ${ }^{1}$ and Sharon C. Glotzer ${ }^{1,2,}$ * \\ ${ }^{1}$ Department of Chemical Engineering, University of Michigan, Ann Arbor, MI 48109-2136, USA \\ ${ }^{2}$ Department of Materials Science and Engineering, \\ University of Michigan, Ann Arbor, MI 48109-2136, USA

\begin{abstract}
Patchy particles are a popular paradigm for the design and synthesis of nanoparticles and colloids
\end{abstract} \\ for self-assembly. In "traditional" patchy particles, anisotropic interactions arising from patterned \\ coatings, functionalized molecules, DNA, and other enthalpic means create the possibility for di- \\ rectional binding of particles into higher-ordered structures. Although the anisotropic geometry \\ of non-spherical particles contributes to the interaction patchiness through van der Waals, electro- \\ static, and other interactions, how particle shape contributes entropically to self-assembly is only \\ now beginning to be understood. It has been recently demonstrated that, for hard shapes, en- \\ tropic forces are directional. A newly proposed theoretical framework that defines and quantifies \\ directional entropic forces demonstrates the anisotropic-that is, patchy-nature of these emergent, \\ attractive forces. Here we introduce the notion of entropically patchy particles as the entropic coun- \\ terpart to enthalpically patchy particles. Using three example "families" of shapes, we judiciously \\ modify entropic patchiness by introducing geometric features to the particles so as to target specific \\ crystal structures, which then assembled with Monte Carlo simulations. We quantify the emergent \\ entropic valence via a potential of mean force and torque. We generalize these shape operations to \\ shape anisotropy dimensions, in analogy with the anisotropy dimensions introduced for enthalpically \\ patchy particles. Our findings demonstrate that entropic patchiness and emergent valence provide a \\ way of engineering directional bonding into nanoparticle systems, whether in the presence or absence \\ of additional, non-entropic forces.
}

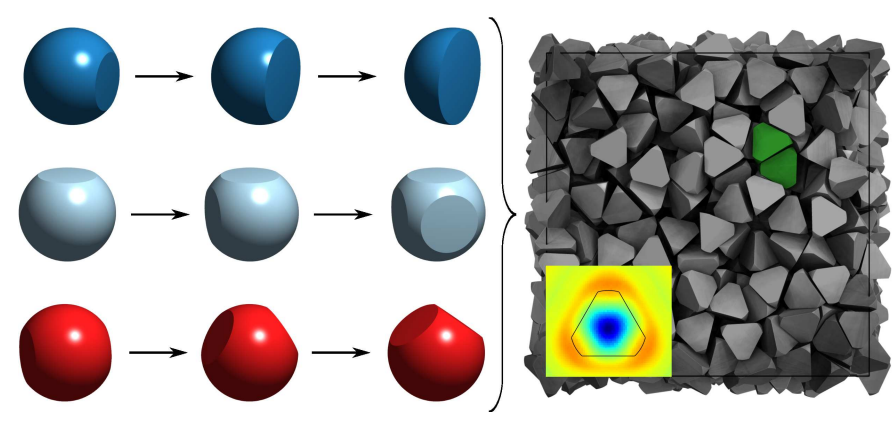

I. INTRODUCTION

Patchy particles 1, 2] self assemble into nanoparticle superlattices and colloidal crystals by exploiting anisotropic interactions arising from, e.g., molecular patterning, DNA functionalization, and charge heterogeneity. Examples of patchy particles include Janus colloids [3] 9, striped nanospheres [10] and nanorods [1], and DNA-coated patchy particles [12, among many others 2 , 13. When the particles are not spheres, non-isotropic van der Waals and other forces also contribute to interaction patchiness. With patchy particles, anisotropically placed patches promoting either specific or nonspecific interactions with patches on other particles induce directional "bonding" between particles of the sort typically attributed to molecular substances. To date, patchy particles have been assembled into numerous structures

\footnotetext{
* sglotzer@umich.edu
}

[14 24, many of them isostructural to their atomic and molecular counterparts.

Recently, there has been considerable focus on the contribution of entropic forces to the assembly of anisotropically shaped particles into complex structures 25 44. A general observation from many of these studies is that dense suspensions of hard, faceted particles align their facets so as to maximize the system entropy, giving rise to ordered structures as complex as colloidal quasicrystals [29, 35] and crystals with unit cells containing as many as 52 particles 36. Damasceno, et al. 35, 36] rationalized this tendency toward facet alignment as the emergence of "directional entropic forces" between hard particles. Directional entropic forces (DEFs) are not intrinsic to the particles, but instead are statistical and emerge from the collective behavior of the entire system upon crowding. The DEF approach to the self-assembly of colloidal cubes, octahedra, rhombic dodecahedra, and tetrahexahedra was recently demonstrated by Young et al. [45.

A theoretical framework for the description and quantification of DEFs was proposed recently [46. Using an effective potential of mean force and torque (PMFT), hard polyhedra, spherocylinders, and hemispheres were shown to exhibit spatially anisotropic probability distributions describing the likely positions of neighboring particles, much like the polyvalent nature of molecules. Unlike in molecular systems, however, here the valence is density dependent and-because it arises statistically from collective behavior-is emergent. We define these emergent valence regions of effective attraction between particles as "entropic patches". These patches can achieve strengths of several to many $k_{\mathrm{B}} T$, aligning complementary geomet- 
rical features just as enthalpically patchy particles align complementary enthalpic features.

Here we introduce the notion of entropically patchy particles as the entropic counterpart to enthalpically patchy particles, and we show how DEFs can be engineered through the systematic alteration of particle shape to target specific self-assembled structures. Using three example families of shapes, we systematically apply certain shape operations to the particles so as modify entropic patchiness to be consistent with target specific crystal structures. In contrast to the charge, chemical, etc. mediation of the interaction between sticky patches on enthalpically patchy particles, attractive entropic patches are features in particle shape that promote local dense packing in thermodynamic equilibrium. We show that we indeed obtain these structures through self assembly with Monte Carlo (MC) simulations. We generalize these shape operations to shape anisotropy dimensions, in analogy with the anisotropy dimensions introduced for patchy particles 2. Our findings demonstrate the utility of the notions of entropic patchiness and emergent valence as an additional way of engineering directional bonding into nanoparticle systems, whether in the presence or absence of additional, non-entropic forces.

\section{BACKGROUND}

The PMFT describing the directional entropic force between a pair of hard particles in a system of identical particles has been derived [46] as

$$
F_{12}\left(\Delta \xi_{12}\right)=-k_{\mathrm{B}} T \log \left(H\left(d\left(\Delta \xi_{12}\right)\right) J\left(\Delta \xi_{12}\right)\right)+\tilde{F}_{12}\left(\Delta \xi_{12}\right)
$$

Here, $\Delta \xi_{12}$ describes the relative position and orientation of the particle pair. $H$ is the Heaviside step function, and $d\left(\Delta \xi_{12}\right)$ is the minimum separation distance of the particle pair in their relative position and orientation, which is negative when the particles overlap, and positive when they do not. $J$ is the Jacobian for the set of invariant coordinates of interest for a particular problem. $\tilde{F}_{12}$ is the Helmholtz free energy available to other particles in the system when the relative position and orientation of the pair is fixed.

From Eq. 11, the PMFT can be seen to arise as a competition between two terms. The term coming from $\tilde{F}_{12}$ is determined by the free energy of the system with the pair fixed. If there is no intrinsic attraction among the particles, then this term will tend to induce a locally denser packing for the particle pair. The other contribution comes from the preference of the pair itself for a particular relative position and orientation. $\tilde{F}_{12}$ will be minimized when the particle pair aligns itself to maximize its local packing density according to the shape of the particles. The features of particle shape that facilitate locally dense packing, therefore, act as the "source" of the emergent attractive DEFs. We refer to these features as entropic patches.

\section{RESULTS AND DISCUSSION}

\section{A. Targeted Self-Assembly Through Emergent Valence}

We design entropic patches to self assemble the following target structures: simple cubic, body-centered cubic, diamond, and dodecagonal quasicrystal. Each of these has been reported in experiments or simulations of patchy spheres or hard polyhedra. In all cases, the local coordination shell at least partially dictates the type of crystal structure that assembles 36. We attempt to create similar local coordination shells through entropic patches engineered by slicing facets into hard spheres. Specifically, we simulate spheres with cubic, octahedral, and tetrahedral faceting to induce the appropriate entropic patchiness, and show that at sufficient crowding the desired valence emerges leading to crystallization of target structures consistent with that particular polyvalent coordination via the organization of successive neighbor shells.

We first target the assembly of a (tetrahedrallycoordinated) diamond lattice by tetrahedrally faceting spheres. The diamond lattice has been assembled in simulation by decorating a sphere with four tetrahedrally coordinated enthalpic patches (sticky spots) [1, 47]. We therefore "slice" four equal sized facets into a sphere at the locations of the faces of a regular tetrahedron. We consider a faceting amount of 0 to be a perfect sphere, and 1 to be a perfect tetrahedron. For concreteness, consider a tetrahedron with vertices at $(1,1,1),(-1,-1,1)$, $(-1,1,-1)$, and $(1,-1,-1)$. A perfect sphere is the intersection of this tetrahedron with a sphere centered about the origin with radius $\frac{1}{\sqrt{3}}$. A perfect tetrahedron is the intersection of this tetrahedron and a sphere with radius $\sqrt{3}$. The radius of the sphere required to generate any amount of faceting $\alpha$ between these limits is given by the formula $\frac{1}{\sqrt{3}}(1-\alpha)+\sqrt{3} \alpha$. We performed MC simulations of monodisperse systems of 1000 such particles at fixed volume, for several choices of $\alpha$ (see Methods for details). We computed the force component of the PMFT as a function of the Cartesian components of the separation vector of the particles in the frame of one of the particles. (A detailed description of the computation and a discussion of possible coordinate systems have been given elsewhere 46].) In Fig. 1 we show that monodisperse, tetrahedrally faceted spheres manifest shape-induced entropic valence (via the PMFT) in dense fluids at 50\% packing fraction. Simulations were performed at faceting amounts between a perfect sphere $(\alpha=0)$ and a perfect tetrahedron $(\alpha=1) \sqrt{1}$ At a faceting amount of 0.6 the particles self-assemble a diamond lattice in MC simulation at a packing fraction of $60 \%$ as shown in Fig. 1.

\footnotetext{
${ }^{1}$ See Table 1 in Supporting Information for actual faceting amounts for this and other particles below.
} 
a
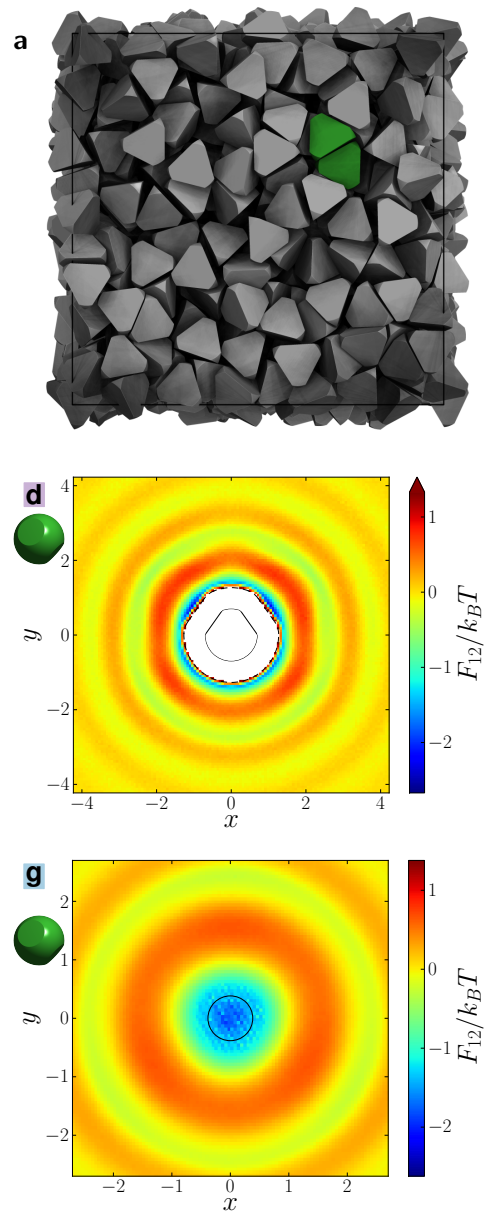

b
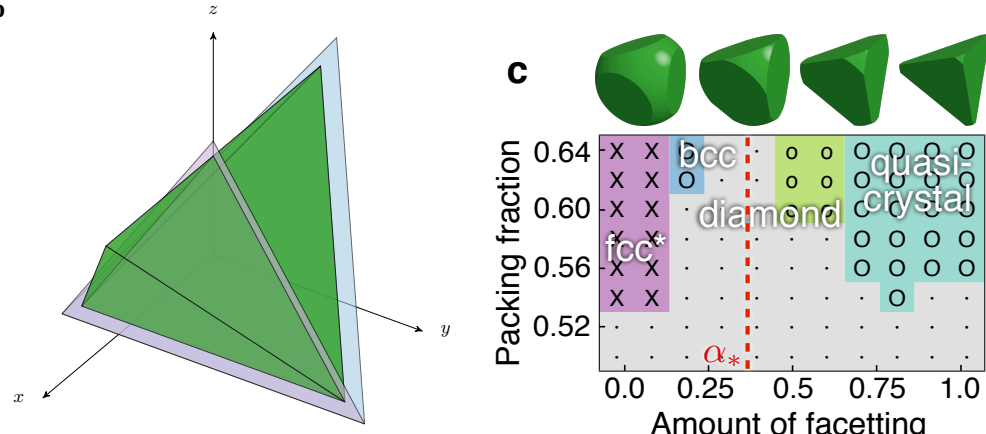

o $0.60-x \quad x \cdot$ diamone ccrystab
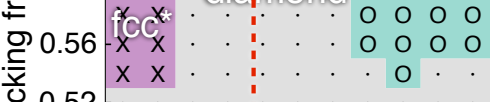

ণั

$\mathrm{X} \times$

$\begin{array}{llll}0 & 0 & 0 & 0\end{array}$

O

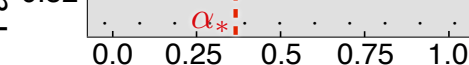

Amount of facetting
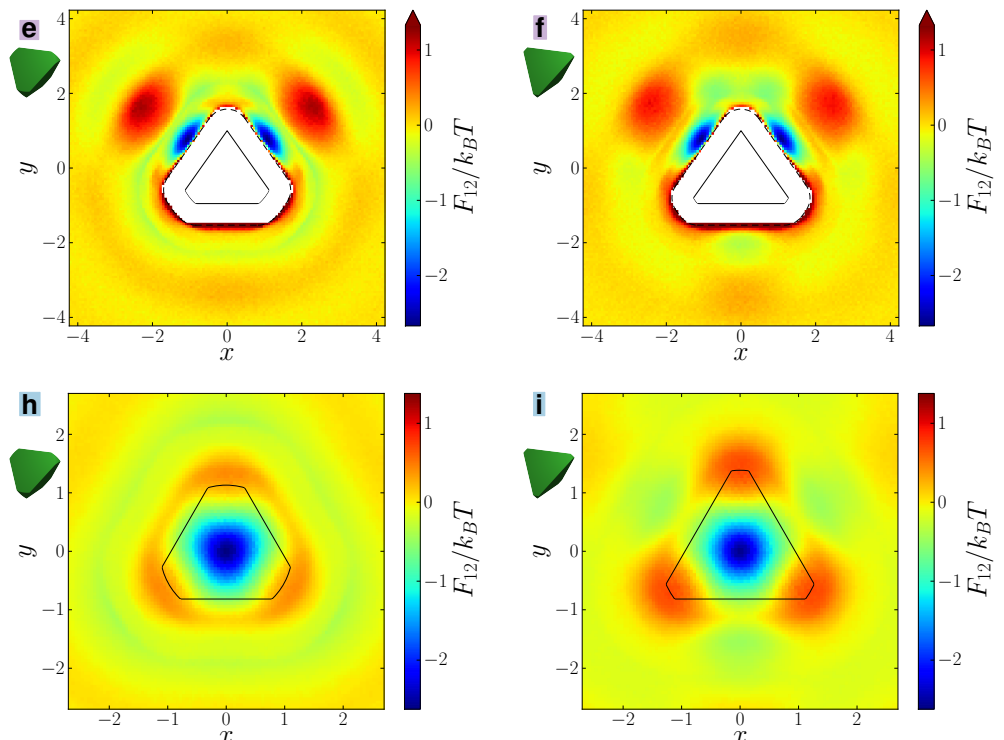

FIG. 1. We demonstrate shape-induced entropic valence in monodisperse systems of hard tetrahedrally faceted spheres by computing the PMFT for a pair of particles in a system of 1000 particles at packing fraction 0.5 (a). Slicing the potential along two different planes (b) shows the induced valence in a plane through the facet ( $\mathrm{d}$, e, and f) and a plane parallel to the facet ( $\mathrm{g}, \mathrm{h}$, and i). At sufficient crowding, the entropic forces arising from shape entropy lead to crystallization (c). As the faceting amount increases from 0.1 ( $\mathrm{d}$ and $\mathrm{g}$ ), to 0.6 (e and $\mathrm{h}$ ), to 0.8 ( $\mathrm{f}$ and $\mathrm{i}$ ), the PMFT shows greater evidence of shape-induced entropic valence that determines the crystal structure, even at insufficient crowding (50\%) to provoke crystallization.

Also note that when the faceting amount $\alpha$ exceeds

$$
\alpha_{*}=\frac{\sqrt{3}-1}{2} \approx 0.3660254
$$

(dashed red line in Fig. 1:) the faceting patches share adjacent edges, which we would expect to have an effect on the local dense packing. Only above this faceting amount are we able to assemble the diamond lattice as shown in Fig. 11. Note that a family of moderately truncated tetrahedra also assemble a diamond lattice[35].

As a second example, we next target simple cubic lattices by slicing cubically coordinated facets into hard spheres. We denote a perfect sphere as 0 and a perfect cube as 1 . For concreteness, take the vertices of the cube to be at $(1,1,1)$, and at similar locations in each of the other octants. A perfect sphere is the intersection of this cube with a sphere centered about the origin with unit radius. A perfect cube would be the intersection with a sphere of radius $\sqrt{3}$. The radius of the sphere required to generate any amount of faceting $\alpha$ in between a cube and a sphere is given by the formula $1+(\sqrt{3}-1) \alpha$. In Fig. 2 we show the role of the PMFT in generating directional entropic forces that cause particles to have high positional correlation at facet locations.

We compute the PMFT in a monodisperse system of hard cubically faceted spheres at a density of $50 \%$ (in the fluid phase) at faceting amounts of $0.2,0.5$, and $0.82^{2}$ We plot the PMFT in panels d-i, by slicing according to the diagram in Fig. $2 \mathrm{~b}$ with faceting amount $\alpha$ increasing from left to right. In panels d-f (label ed also with blue), we show slices of the PMFT at constant $z$ as depicted by the blue plane in Fig. $2 \mathrm{~b}$. (Here we take $z \approx 0$.) In panels g-i (labelled also with mauve) we slice the PMFT

\footnotetext{
2 See supporting information for other faceting amounts.
} 
a

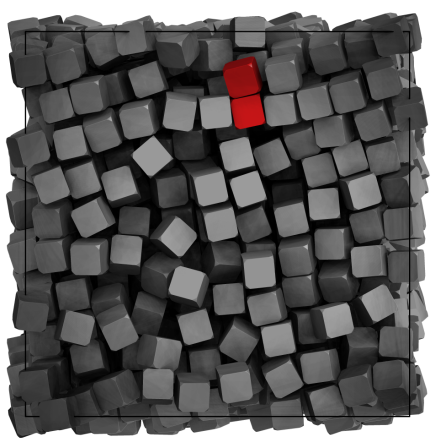

b
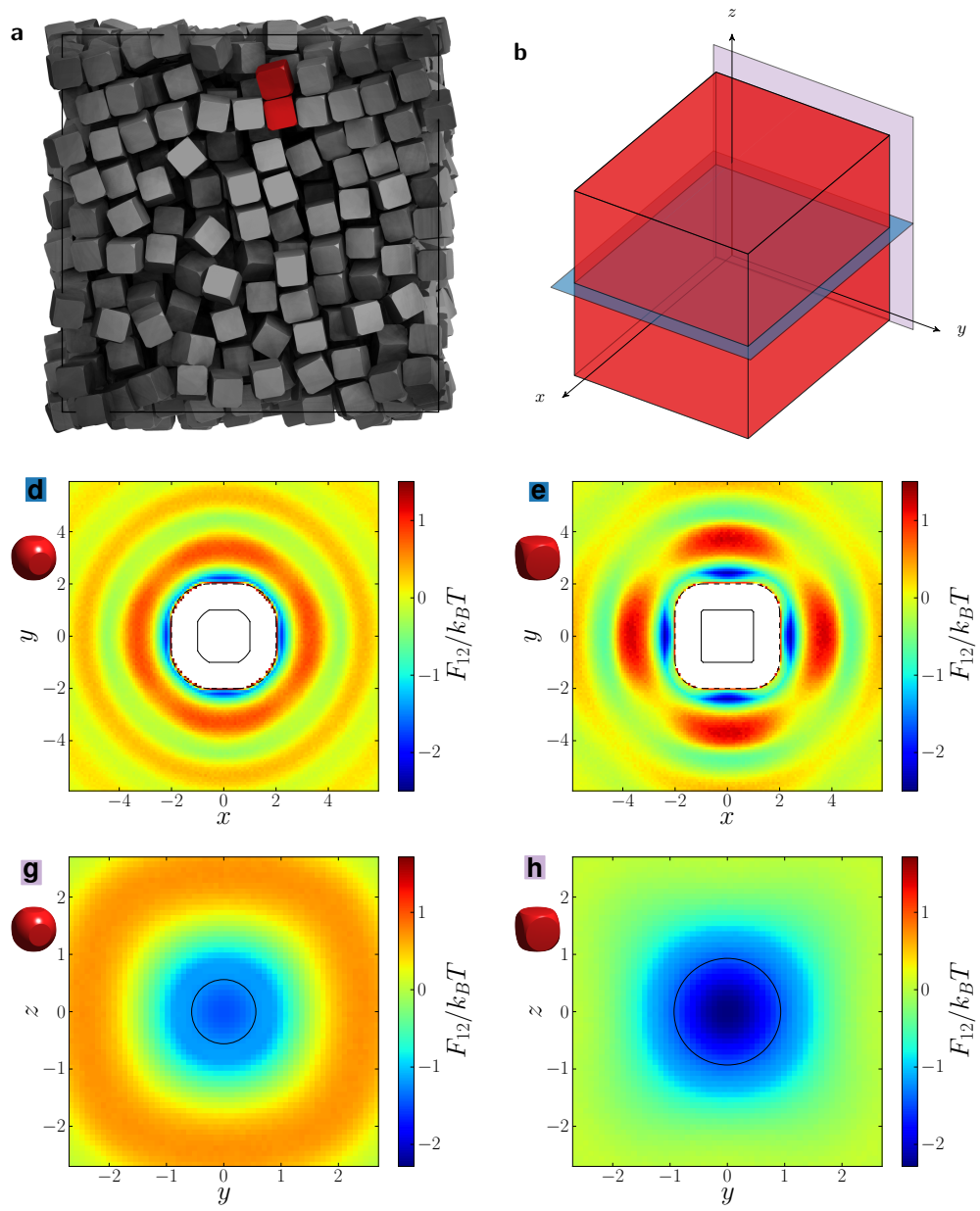
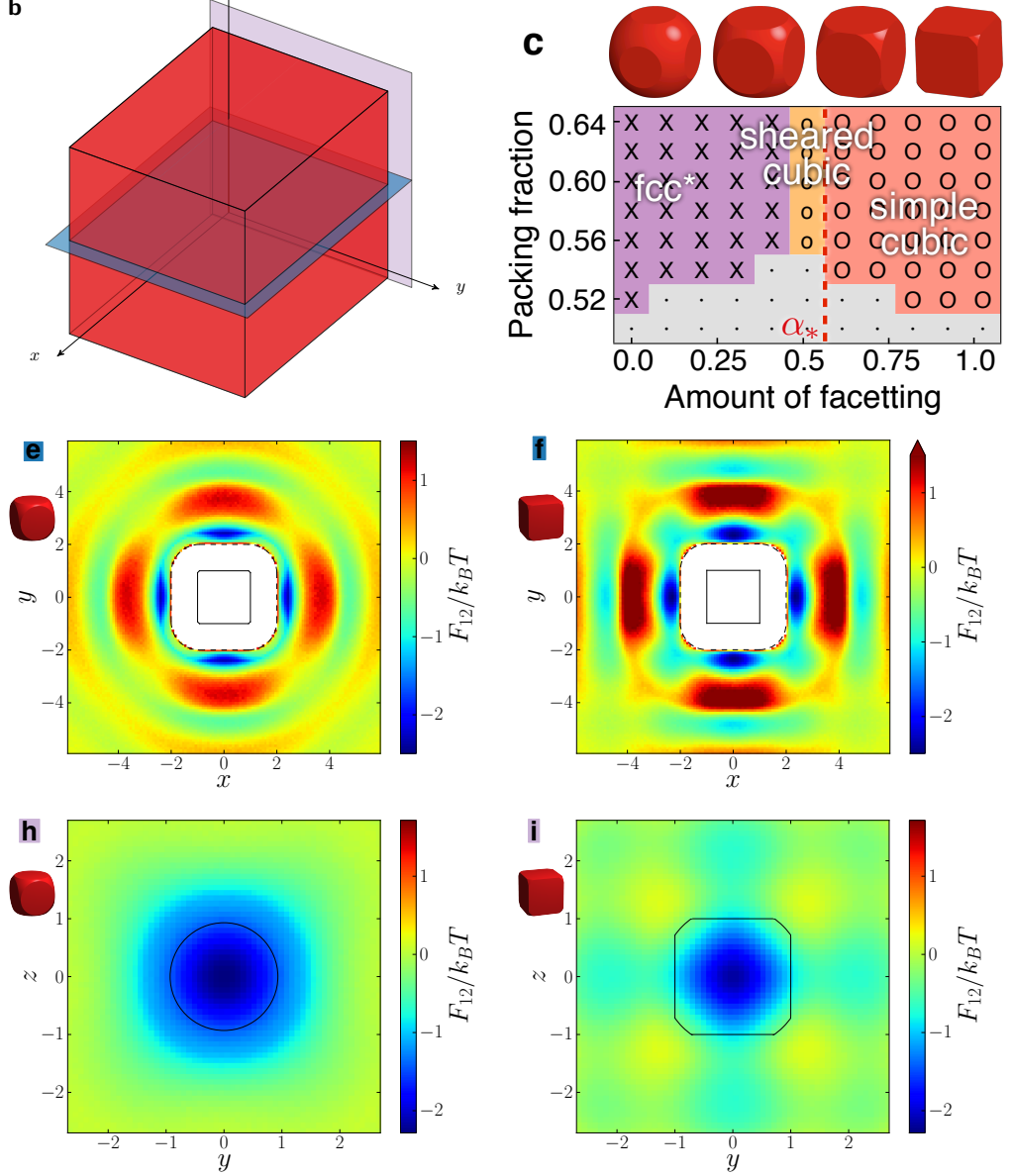

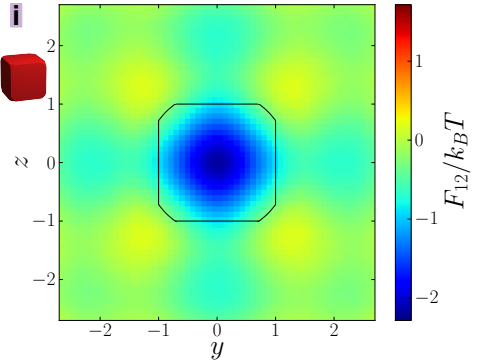

FIG. 2. We demonstrate shape-induced entropic valence in monodisperse systems of hard cubically faceted spheres by computing the PMFT for a pair of particles in a system of 1000 particles at packing fraction 0.5 (a). Slicing the potential along two different planes (b) shows the induced valence in a plane through the facet (d, e, and f) and a plane parallel to the facet ( $g$, h, and i). At sufficient crowding, the entropic forces arising from shape entropy lead to crystallization (c). As the faceting amount increases from 0.2 ( $\mathrm{d}$ and $\mathrm{g}$ ), to 0.5 (e and $\mathrm{h}$ ), to 0.8 ( $\mathrm{f}$ and $\mathrm{i}$ ), the PMFT shows greater evidence of shape-induced entropic valence that determines the crystal structure, even at insufficient crowding $(50 \%)$ to provoke crystallization.

parallel to the faceting patch (the mauve plane in Fig. $2 \mathrm{~b}$ ) through the minimum of the potential. We see that upon increasing the faceting amount we induce a greater amount of cubic coordination in the fluid at fixed density. At a faceting amount of 0.2 (panels $d$ and $g$ ), the PMFT is nearly isotropic, indicating that faceting plays only a small role in locally ordering the particles. However, at faceting amounts of 0.5 (panels e and h) and 0.8 (panels $\mathrm{f}$ and i) we observe PMFT differences on the order of $2-$ $3 k_{\mathrm{B}} T$ favoring alignment of facets. Simple cubic lattices (Fig. 2k) assemble at $\alpha \approx 0.6$ or more in MC simulations at packing fractions of $54 \%$ or more. Note that when the faceting amount $\alpha$ exceeds

$$
\alpha_{*}=\frac{\sqrt{2}-1}{\sqrt{3}-1} \approx 0.565826,
$$

(dashed red line in Fig. 2r) the shape of the faceting patch goes from being circular to having four straight edges that are shared by adjacent patches. The existence of this edge should have an effect on the geometry of the locally preferred packing, and it is, perhaps, not surprising that above this faceting amount we observe the assembly of simple cubic lattices. The self-assembly of cubic, or nearly cubic particles into simple cubic lattices has been seen before [38, 41].

As a final example, we target a bcc or sheared bcc crystal with eight nearest neighbors by octahedrally faceting a sphere. We denote a perfect sphere as 0 and a perfect octahedron as 1 . For concreteness, take the vertices to lie at a unit distance from the origin along each of the Cartesian coordinate axes, at $(1,0,0),(-1,0,0)$, etc. A perfect sphere is the intersection of this cube with a sphere centered about the origin with radius $\frac{1}{\sqrt{3}}$. A perfect octahedron is the intersection with a sphere of unit radius. The radius of the sphere required to generate any amount of faceting $\alpha$ between the sphere and the octahe- 
a

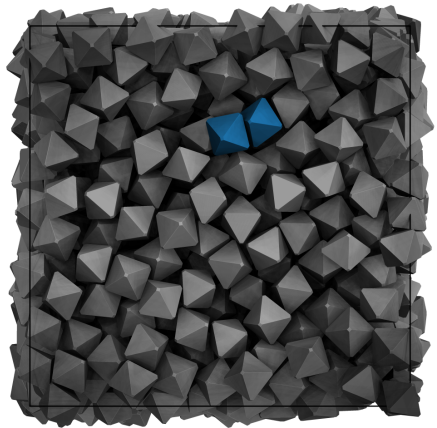

b
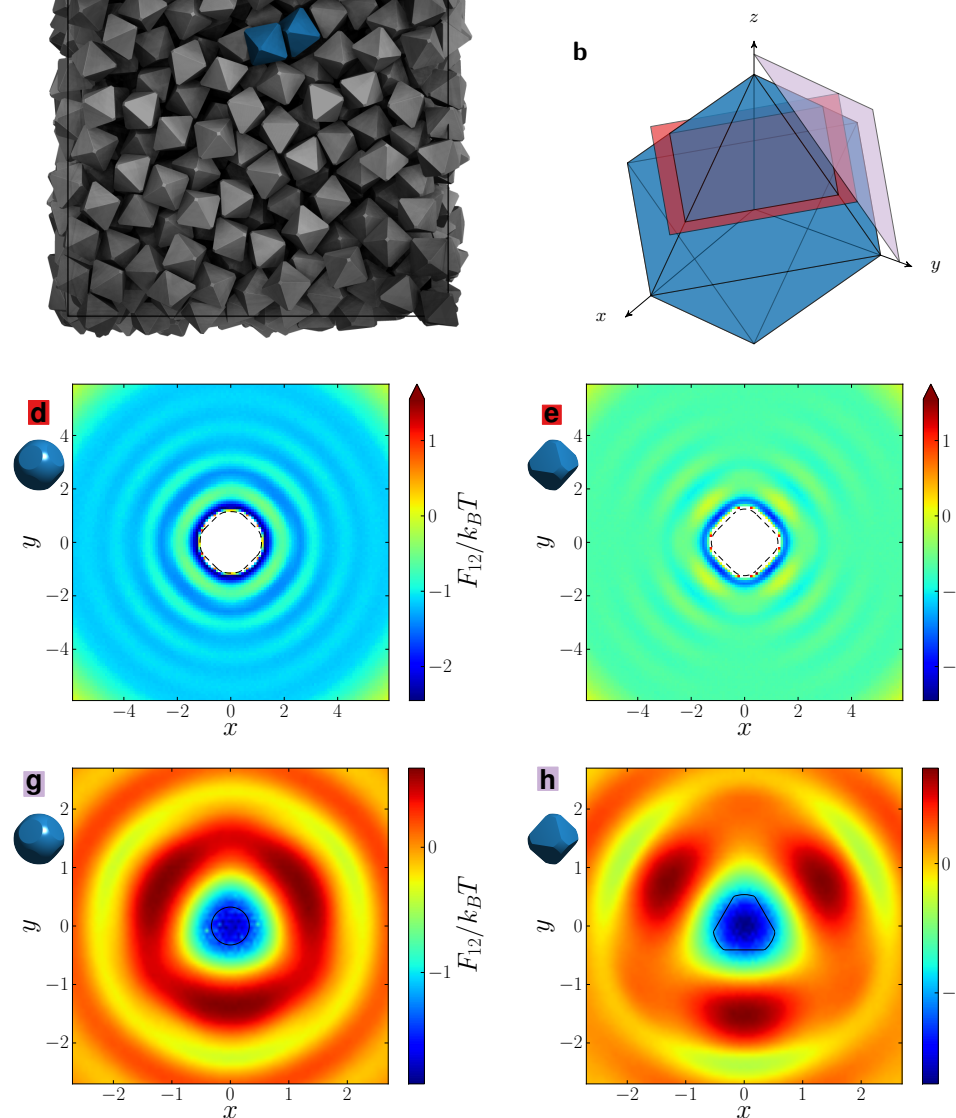

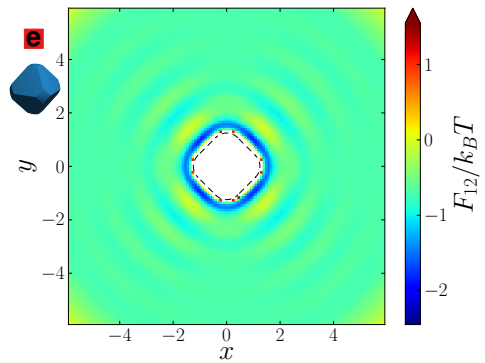

c
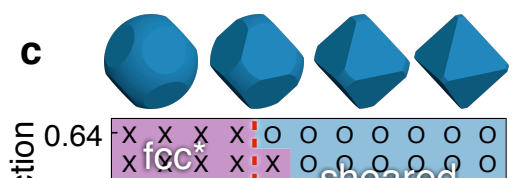

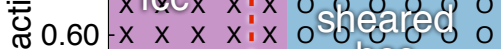

$\begin{array}{lllllllllllll}x & x & x & x & x & 0 & 0 & 0.56 & 0 & 0\end{array}$

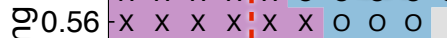

\begin{tabular}{llllllllllllll}
\hline & $x$ & $x$ & $x$ & $x$ & $x$ & $x$ & 0 & 0 & 0
\end{tabular} तु 0.52

$\begin{array}{lllll}0.0 & 0.25 & 0.5 & 0.75 & 1.0\end{array}$

Amount of facetting
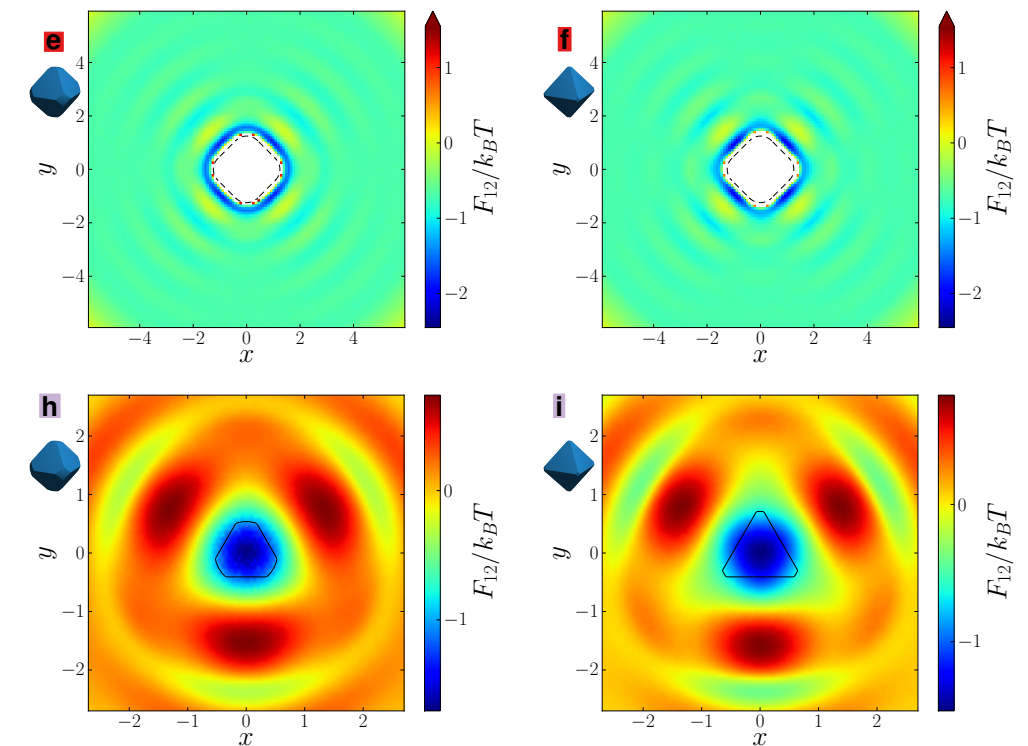

FIG. 3. We demonstrate shape-induced entropic valence in monodisperse systems of hard octahedrally faceted spheres by computing the PMFT for a pair of particles in a system of 1000 particles at packing fraction 0.5 (a). Slicing the potential along two different planes (b) shows the induced valence in a plane through the facet (d, e, and f) and a plane parallel to the facet ( $g, h$, and i). At sufficient crowding, the entropic forces arising from shape entropy lead to crystallization (c). As the faceting amount increases from 0.2 ( $\mathrm{d}$ and g), to 0.6 (e and h), to 0.8 (f and i), the PMFT shows greater evidence of shape-induced entropic valence that determines the crystal structure, even at insufficient crowding (50\%) to provoke crystallization.

dron is given by the formula $1+\left(1-\frac{1}{\sqrt{3}}\right) \alpha$. Simulations were performed at faceting amounts between these two limits. At low faceting amounts, where the entropic valence (patchiness) is fairly isotropic, the systems assembled fcc crystals. However, at faceting amounts as low as 0.4 , we observe sheared bcc crystals. Note that when the faceting amount $\alpha$ exceeds

$$
\alpha_{*}=\frac{\frac{\sqrt{3}}{\sqrt{2}}-1}{\sqrt{3}-1} \approx 0.307007,
$$

(dashed red line in Fig. 3r) the faceting patches begin to share adjacent edges. This figure coincides with the lowest faceting amount at which we observed the sheared bcc crystal in our simulations. Octahedra have been studied previously, [35-38, 43] where bcc and sheared bcc lattices were also observed. One interesting feature of the octahedrally faceted particles is that entropically preferred local dense packings have particles situated face- to-face, but with the orientation of the adjacent faces rotated by $180^{\circ}$. In the resulting "star of David" arrangement (see Fig. S10) the protruding vertices reduce the free volume available to the surrounding particles. This means that although face-to-face arrangements are still favored by shape entropy, as exhibited in the increasing anisotropy of the PMFT depicted in Fig. 3, the strength of the entropic patch is actually lessened at high degrees of faceting, compared with lower degrees.

\section{B. Generalization and Anisotropy Dimensions}

The angular specificity achieved by facet alignment via DEFs is reminiscent of the angular specificity of enthalpic interactions conceptualized within the patchy particle paradigm 1, 2. However, in contrast to the chemical or other patterning that leads to enthalpic patchiness, the 
(a) patch size

(b) curvature radius
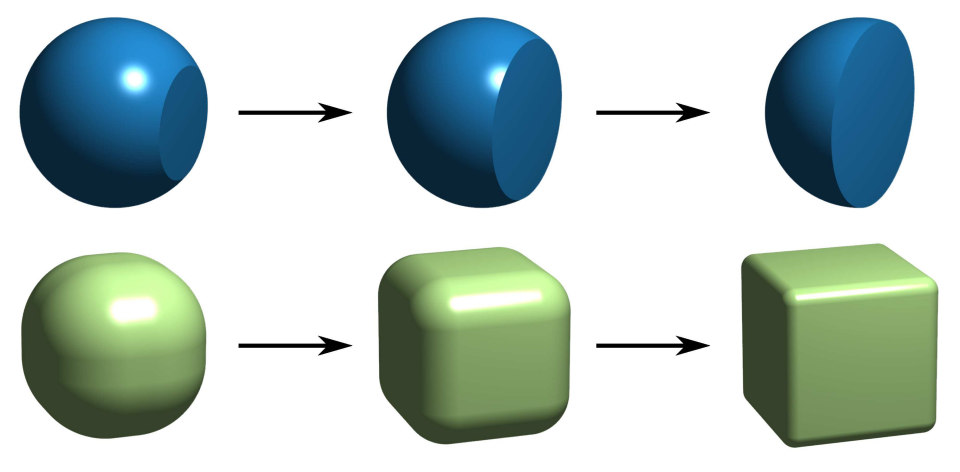

(c) aspect ratio

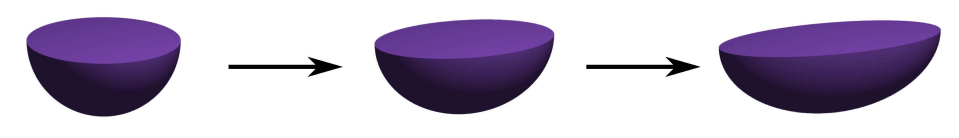

(d) patch angle

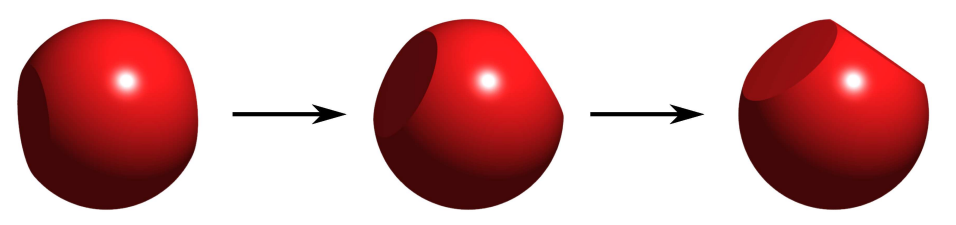

(e) number of patches

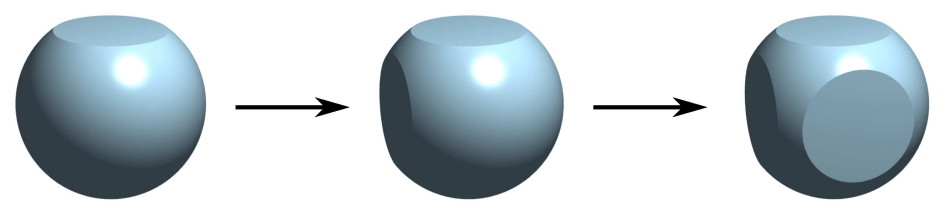

(f) shape composition operation

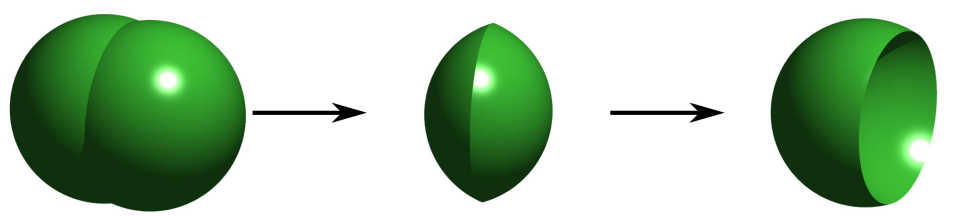

(g) shape gradient

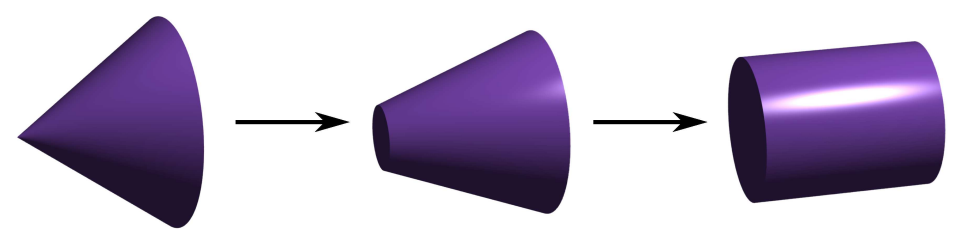

(h) roughness

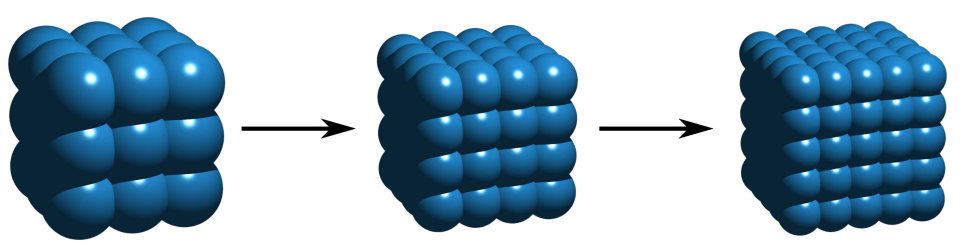

FIG. 4. (a) The creation and altering of entropic patches can be conceptualized as anisotropy dimensions: (a) patch size, (b) curvature radius, (c) aspect ratio, and (d) patch angle, (e) number of patches, (f) shape composition operation, (g) shape gradient, and (h) roughness.

angular specificity of interactions arises here solely due to entropic considerations arising from features in particle shape that promote local dense packing. These features act as "entropic patches" that cause preferential alignment. Beyond the simple faceting of hard spheres, there are many ways of altering particle shape to introduce patches that promote local dense packing.

The various shape operations that may be applied to generate attractive entropic patches may be described in terms of anisotropy dimensions (Fig. 4), as was done for enthalpic patches 2. Of course, in contrast to traditional sticky patches, entropic patchiness is an emergent, effective concept that depends not only on density but also on all geometric features within the characteristic length of the interaction. Such features may include, e.g., flat facets or other low curvature regions, and interlocking 


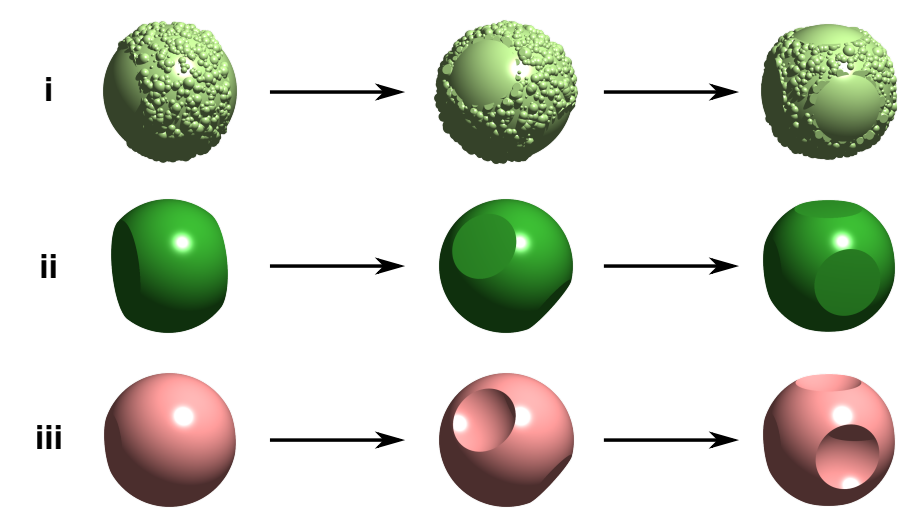

FIG. 5. Schematic showing the the same anisotropy dimension (e, number of patches) applied to three different particle types: (i) a roughened colloid, (ii) a faceted sphere, and (iii) a dimpled sphere.

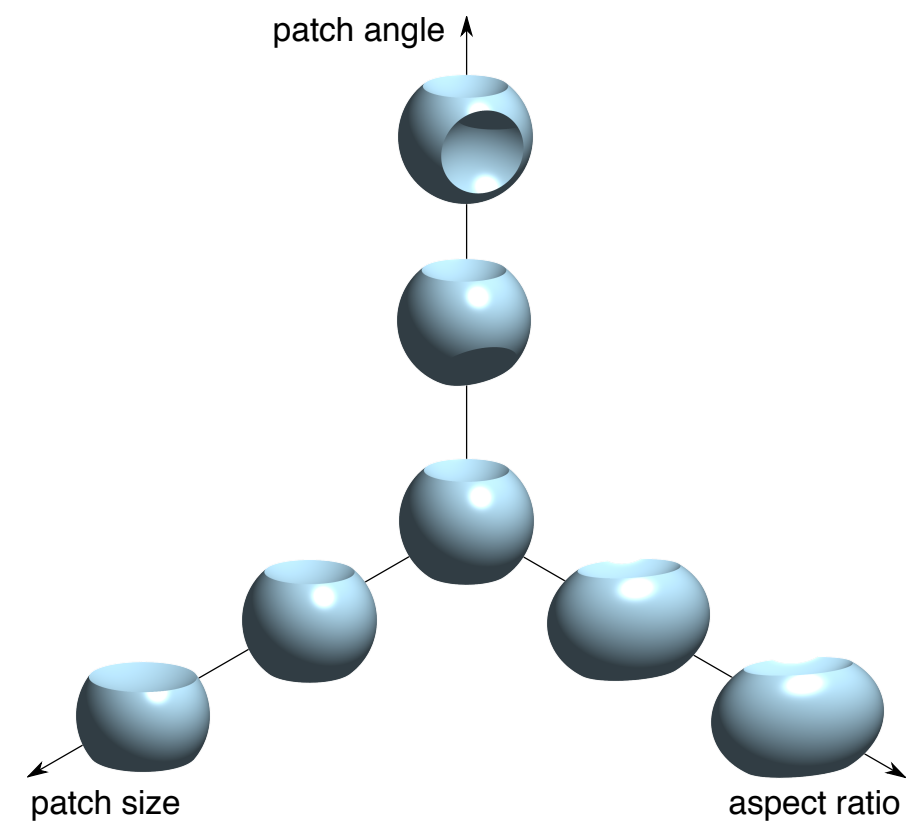

FIG. 6. Schematic showing the result of combining various anisotropy dimensions by applying patch angle (d), aspect ratio (c), and patch size (a) from Fig. 4 to a sphere with two "dimple" patches.

or "mating" features. Conversely, high curvature regions could be used to introduce repulsive patches.

Eight examples of shape anisotropy dimensions are illustrated in Fig. 4. Many of these anisotropy dimensions have already been explored in particles synthesized in the literature. For example, in lock-and-key colloids [50] the anisotropy dimensions of patch size (a), curvature radius (b), and shape composition operation (f) have been synthesized [51. In roughened colloids the anisotropy dimensions of patch size (a), aspect ratio (c), patch angle (d), number of patches (e) and roughness (h) have been synthesized [52 58]. There are many other examples of work in the literature that can be considered explorations of these anisotropy dimensions [45, 59, 88.

Shape anisotropy dimensions can be combined in different ways to yield various particles that have appeared in the literature, e.g. lock-and-key colloids [50, 89], and various novel particle geometries. For example, in Fig. 5 we consider applying the anisotropy dimension of number of patches to three different species of particle: roughened colloids 52 58, faceted spheres, and dimpled spheres [50, 51, 88]. In Fig. 6 we consider the shape space defined by the application of three orthogonal anisotropy dimensions to the dimpled sphere. We also note that many of these anisotropy dimensions map naturally to anisotropy dimensions introduced for enthalpically patchy particles 2. Examples of enthalpically patchy particles and their entropically patchy analogues are shown in Fig. 77a. As depicted in Fig. 7b, combining both enthalpic and entropic patchiness provides opportunities for enhancing particular desired particle alignment, or produce competing forces that may give rise to structures of high complexity.

As a final note, we point out that entropically patchy 

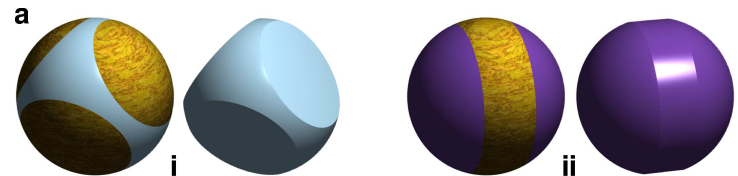

b

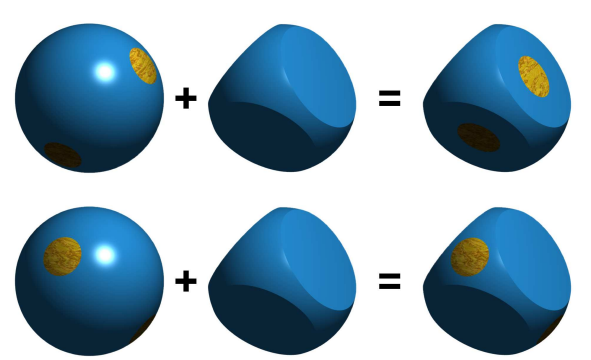

FIG. 7. Panel (a): Many anisotropy dimensions for enthalpically patchy particles 2] have entropically patchy counterparts. Example traditional sticky patchy particles left (i) and (ii) with entropically patchy counterparts right (i) and (ii). Panel (b): Enthalpic patches can be combined with entropic patches to enhance or inhibit entropic patchiness as shown schematically here. This can be obtained by using the same direction for both types of patches (top), or by using different directions for the entropic patches and the enthalpic patches (bottom).

particles are also relevant in systems with depletants, since depletant-induced colloidal crystallization is controlled by the same entropic mechanism as the crystallization of hard colloids. 46] The interaction range for DEFs is determined by the scale of the particles being integrated out in the calculation of the PMFT. In traditional depletion systems, the depletants are typically much smaller than the colloids and penetrable. This hierarchy in scales produces a very short ranged interaction between nanoparticles or colloids, which allows for a clearer separation between geometric features on the colloids that contribute to local dense packing, and thereby eases the identification of the features that are the entropic patches. Particularly salient examples of entropically patchy particles assembled through depletion forces include the work on selectively roughened colloids [52 58, lock-and-key colloids [50, 51, 88 91, and polyhedrally-shaped metal nanoparticles 37 , 45, each of which exploits geometrical features to create anisotropy in entropic interactions. These and related works have been reviewed elsewhere 92 .

\section{CONCLUSION}

We showed in three example model systems that by judiciously engineering particle shape we can induce angularly specific interactions between hard particles strong enough and directional enough to induce the selfassembly of a targeted crystal structure. The DEFs responsible for this ordering can be measured experimentally using, e.g., optical tweezers and existing confocal microscopy techniques [78, 93. We abstracted these examples of particle design to be part of a much broader method of shape engineering through entropic patches that serves to provide a means of self-assembling materials through entropic interactions alone. We introduced anisotropy dimensions to exhibit and organize the various ways in which particle shape can be engineered to exploit this method of realizing complex structures.

When combined with traditional enthalpic patchiness, entropic patchiness greatly enlarges the already vast design space for new nano- and micron-scale building blocks. The theoretical framework based upon the PMFT 46] allows the quantitative assessment of the relative strength of entropic driving forces for assembly when other forces also contribute. At the nanoscale, particles are seldom solely hard 94. However, the fact that DEFs cause attractive interactions on the order of a few $k_{\mathrm{B}} T$ at intermediate packing densities that are easily experimentally accessible suggests there might be a large class of systems in which electrostatic or other forces can be sufficiently controlled such that entropic patches supply the dominant force controlling their self-assembly, as was demonstrated recently [45, 95, 96. When enthalpic interactions tend to drive facet alignment anyway, as in, e.g., ligand-coated faceted nano particles [96, 97, the entropic contribution will only enhance that tendency.

\section{METHODS}

To measure the PMFT we performed MC simulations of dense fluids of 1000 hard faceted spheres at fixed volume. We note [46] that the PMFT can be defined implicitly from the partition function

$$
\mathcal{Z}=\int d\left(\Delta \xi_{12}\right) e^{-\beta F_{12}\left(\Delta \xi_{12}\right)}
$$

In this form the PMFT can be seen to be the logarithm of the integrand of the partition function. We computed the PMFT by examining the displacement between particles, which we computed in the coordinate frame of each particle, and partitioned into defined regions. The orientations of the second particle were integrated over so as to sufficiently reduce the dimensionality of the potential to allow direct visualization. The possible relative positions of the particles were subdivided into a number of regions, and the PMFT was computed by observing the relative frequency of observing a pair of particles in each of these regions, as indicated in Eq. (5). Errors quoted are standard errors of the mean of independent runs of independently equilibrated systems. Full details on the source and analysis of numerical errors that can arise in computing DEFs can be found elsewhere [46].

The MC method employed above, and for the results shown in Figs. 1. 2, and 3 employed single particle moves for both translation and orientation. In all cases the simulation box was taken to be periodic and the volume 
fixed. However, for Figs. 11; , 2k, and 3r, the box was permitted to shear at fixed volume. Overlaps were checked using the same implementation of the GJK algorithm 98 used other work by some of the present authors 36 .

This document is the unedited Author's version of a Submitted Work that was subsequently accepted for publication in ACS Nano, copyright (c) American Chemical Society after peer review. To access the final edited and published work see DOI:10.1021/nn4057353

\section{ACKNOWLEDGMENTS}

We thank D. Klotsa and B. Schultz for helpful suggestions. This material is based upon work supported by, or in part by, the U.S. Army Research Office under Grant Award No. W911NF-10-1-0518, the DOD/ASD(R\&E) under Award No. N00244-09-1-0062, and the Biomolecular Materials Program of the Materials Engineering and Science Division of Basic Energy Sciences at the U.S. Department of Energy under Grant No. DE-FG0202ER46000. The calculations of the hard particle PMFT were supported by ARO. The development of the PMFT and anisotropy dimensions were supported by DoE. Any opinions, findings, and conclusions or recommendations expressed in this publication are those of the author(s) and do not necessarily reflect the views of the $\mathrm{DOD} / \mathrm{ASD}(\mathrm{R} \& \mathrm{E})$.
[1] Z. Zhang and S. C. Glotzer, Nano Lett. 4, 1407 (2004).

[2] S. C. Glotzer and M. J. Solomon, Nat. Mater. 6, 557 (2007)

[3] V. Paunov and O. Cayre, Adv. Mater. 16, 788 (2004).

[4] O. Cayre, V. N. Paunov, and O. D. Velev, J. Mater. Chem. 13, 2445 (2003)

[5] K.-H. Roh, D. C. Martin, and J. Lahann, Nat. Mater. 4, $759(2005)$

[6] G. Zhang, D. Wang, and H. Möhwald, Nano Lett. 5, 143 (2005)

[7] J.-Q. Cui and I. Kretzschmar, Langmuir 22, 8281 (2006)

[8] L. Hong, S. Jiang, and S. Granick, Langmuir 22, 9495 (2006)

[9] Q. Zhang, Y. H. Lee, I. Y. Phang, S. Pedireddy, W. W. Tjiu, and X. Y. Ling, Langmuir 29, 12844 (2013)

[10] A. M. Jackson, J. W. Myerson, and F. Stellacci, Nat. Mater. 3, 330 (2004)

[11] B. R. Martin, D. J. Dermody, B. D. Reiss, M. Fang, L. A. Lyon, M. J. Natan, and T. E. Mallouk, Adv. Mater. 11, 1021 (1999).

[12] Y. Wang, Y. Wang, D. R. Breed, V. N. Manoharan, L. Feng, A. D. Hollingsworth, M. Weck, and D. J. Pine, Nature 491, 51 (2012).

[13] A. B. Pawar and I. Kretzschmar, Macromol. Rapid Commun. 31, 150 (2010).

[14] S. Park, J.-H. Lim, S.-W. Chung, and C. A. Mirkin, Science 303, 348 (2004)

[15] F. Li, W. C. Yoo, M. B. Beernink, and A. Stein, J. Am. Chem. Soc. 131, 18548 (2009), http://pubs.acs.org/doi/pdf/10.1021/ja908364k.

[16] H. Onoe, K. Matsumoto, and I. Shimoyama, Small 3, $1383(2007)$

[17] A. Figuerola, I. R. Franchini, A. Fiore, R. Mastria, A. Falqui, G. Bertoni, S. Bals, G. Van Tendeloo, S. Kudera, R. Cingolani, and L. Manna, Adv. Mater. 21, 550 (2009).

[18] M. Rycenga, J. M. McLellan, and Y. Xia, Adv. Mater. 20, $2416(2008)$

[19] G. Zhang, D. Wang, and H. Möhwald, Angew. Chem., Int. Ed. 44, 7767 (2005)

[20] S. Gangwal, A. Pawar, I. Kretzschmar, and O. D. Velev, Soft Matter 6, 1413 (2010)

[21] Q. Chen, S. C. Bae, and S. Granick, Nature 469, 381
(2011)

[22] Q. Chen, J. K. Whitmer, S. Jiang, S. C. Bae, E. Luijten, and S. Granick, Science 331, 199 (2011)

[23] Z. Mao, H. Xu, and D. Wang, Adv. Funct. Mater. 20, $1053(2010)$

[24] F. Li, D. P. Josephson, and A. Stein, Angew. Chem., Int. Ed. 50, 360 (2011)

[25] D. Frenkel, J. Phys. Chem. 91, 4912 (1987)

[26] A. Stroobants, H. N. W. Lekkerkerker, and D. Frenkel, Phys. Rev. A 36, 2929 (1987).

[27] T. Schilling, S. Pronk, B. Mulder, and D. Frenkel, Phys. Rev. E 71, 036138 (2005).

[28] B. S. John, C. Juhlin, and F. A. Escobedo, J. Chem. Phys. 128, 044909 (2008)

[29] A. Haji-Akbari, M. Engel, A. S. Keys, X. Zheng, R. G. Petschek, P. Palffy-Muhoray, and S. C. Glotzer, Nature 462, 773 (2009), arXiv:1012.5138 [cond-mat.soft],

[30] W. H. Evers, B. D. Nijs, L. Filion, S. Castillo, M. Dijkstra, and D. Vanmaekelbergh, Nano Lett. 10, 4235 (2010)

[31] U. Agarwal and F. A. Escobedo, Nat. Mater. 10, 230 (2011).

[32] K. Zhao, R. Bruinsma, and T. G. Mason, Proc. Natl. Acad. Sci. U.S.A. 108, 2684 (2011).

[33] A. Haji-Akbari, M. Engel, and S. C. Glotzer, Phys. Rev. Lett. 107, 215702 (2011), arXiv:1106.5561 [condmat.stat-mech]

[34] L. Rossi, S. Sacanna, W. T. M. Irvine, P. M. Chaikin, D. J. Pine, and A. P. Philipse, Soft Matter 7, 4139 (2011)

[35] P. F. Damasceno, M. Engel, and S. C. Glotzer, ACS Nano 6, 609 (2012), arXiv:1109.1323 [cond-mat.soft]

[36] P. F. Damasceno, M. Engel, and S. C. Glotzer, Science 337, 453 (2012), arXiv:1202.2177 [cond-mat.soft],

[37] J. Henzie, M. Grünwald, A. Widmer-Cooper, P. L. Geissler, and P. Yang, Nat. Mater. 11, 131 (2012).

[38] R. Ni, A. P. Gantapara, J. de Graaf, R. van Roij, and M. Dijkstra, Soft Matter 8, 8826 (2012), arXiv:1111.4357 [cond-mat.soft]

[39] J. Zhang, Z. Luo, B. Martens, Z. Quan, A. Kumbhar, N. Porter, Y. Wang, D.-M. Smilgies, and J. Fang, J. Am. Chem. Soc. 134, 14043 (2012)

[40] U. Agarwal and F. A. Escobedo, J. Chem. Phys. 137, 
024905 (2012)

[41] F. Smallenburg, L. Filion, M. Marechal, and M. Dijkstra, Proc. Natl. Acad. Sci. U.S.A. 109, 17886 (2012) arXiv:1111.3466 [cond-mat.soft]

[42] M. Marechal, A. Patti, M. Dennison, and M. Dijkstra, Phys. Rev. Lett. 108, 206101 (2012), arXiv:1112.1209 [cond-mat.soft].

[43] A. P. Gantapara, J. de Graaf, R. van Roij, and M. Dijkstra, Phys. Rev. Lett. 111, 015501 (2013).

[44] L. Cademartiri, K. J. M. Bishop, P. W. Snyder, and G. A. Ozin, Philos. Trans. R. Soc., A 370, 2824 (2012).

[45] K. L. Young, M. L. Personick, M. Engel, P. F. Damasceno, S. N. Barnaby, R. Bleher, T. Li, S. C. Glotzer, B. Lee, and C. A. Mirkin, Angew. Chem., Int. Ed. in press (2013), 10.1002/anie.201306009

[46] G. van Anders, N. K. Ahmed, D. Klotsa, M. Engel, and S. C. Glotzer, (2013), arXiv:1309.1187, arXiv:1309.1187 [cond-mat.soft].

[47] F. Romano, E. Sanz, and F. Sciortino, J. Phys. Chem. B. 113, 15133 (2009).

[48] See Table 1 in Supporting Information for actual faceting amounts for this and other particles below.

[49] See supporting information for other faceting amounts.

[50] S. Sacanna, W. T. M. Irvine, P. M. Chaikin, and D. Pine, Nature 464, 575 (2010)

[51] S. Sacanna, M. Korpics, K. Rodriguez, L. ColonMelendez, S.-H. Kim, D. J. Pine, and G.-R. Yi, Nat. Commun. 4, 1688 (2013)

[52] K. Zhao and T. G. Mason, Phys. Rev. Lett. 99, 268301 (2007)

[53] K. Zhao and T. G. Mason, Phys. Rev. Lett. 101, 148301 (2008)

[54] S. Badaire, C. Cottin-Bizonne, J. W. Woody, A. Yang, and A. D. Stroock, J. Am. Chem. Soc. 129, 40 (2007).

[55] S. Badaire, C. Cottin-Bizonne, and A. D. Stroock, Langmuir 24, 11451 (2008)

[56] A. M. Yake, C. E. Snyder, and D. Velegol, Langmuir 23, $9069(2007)$

[57] C. E. Snyder, M. Ong, and D. Velegol, Soft Matter 5, 1263 (2009).

[58] D. J. Kraft, R. Ni, F. Smallenburg, M. Hermes, K. Yoon, D. A. Weitz, A. van Blaaderen, J. Groenewold, M. Dijkstra, and W. K. Kegel, Proc. Natl. Acad. Sci. U.S.A. 109, 10787 (2012)

[59] T. S. Ahmadi, Z. L. Wang, T. C. Green, A. Henglein, and M. A. El-Sayed, Science 272, 1924 (1996).

[60] T. K. Sau and C. J. Murphy, J. Am. Chem. Soc. 126, 8648 (2004)

[61] A. Demortière, P. Launois, N. Goubet, P.-A. Albouy, and C. Petit, J. Phys. Chem. B 112, 14583 (2008).

[62] D. Seo, J. C. Park, and H. Song, J. Am. Chem. Soc. 128, $14863(2006)$

[63] F.-R. Fan, D.-Y. Liu, Y.-F. Wu, S. Duan, Z.-X. Xie, Z.Y. Jiang, and Z.-Q. Tian, J. Am. Chem. Soc. 130, 6949 (2008)

[64] S. E. Habas, H. Lee, V. Radmilovic, G. A. Somorjai, and P. Yang, Nat. Mater. 6, 692 (2007).

[65] H. Song, F. Kim, S. Connor, G. A. Somorjai, and P. Yang, J. Phys. Chem. B 109, 188 (2005).

[66] S. E. Skrabalak, L. Au, X. Li, and Y. Xia, Nat. Protoc. , $2182(2007)$

[67] N. R. Jana, L. Gearheart, and C. J. Murphy, Chem. Commun. , 617 (2001)

[68] F. Kim, S. Connor, H. Song, T. Kuykendall, and
P. Yang, Angew. Chem., Int. Ed. 43, 3673 (2004).

[69] R. Klajn, A. Pinchuk, G. Schatz, and B. Grzybowski, Angew. Chem., Int. Ed. 46, 8363 (2007)

[70] N. Tian, Z.-Y. Zhou, S.-G. Sun, Y. Ding, and Z. L. Wang, Science 316, 732 (2007).

[71] C. J. Murphy, T. K. Sau, A. M. Gole, C. J. Orendorff, J. Gao, L. Gou, S. E. Hunyadi, and T. Li, J. Phys. Chem. B 109, 13857 (2005).

[72 M. Grzelczak, J. Perez-Juste, P. Mulvaney, and L. M. Liz-Marzan, Chem. Soc. Rev. 37, 1783 (2008)

[73] N. Zhao, W. Ma, Z. Cui, W. Song, C. Xu, and M. Gao, ACS Nano 3, 1775 (2009).

[74] C. Ho, A. Keller, J. Odell, and R. Ottewill, Colloid Polym. Sci. 271, 469 (1993)

[75 T. D. Clark, J. Tien, D. C. Duffy, K. E. Paul, and G. M. Whitesides, J. Am. Chem. Soc. 123, 7677 (2001) http://pubs.acs.org/doi/pdf/10.1021/ja010634l

[76] K. P. Velikov, T. van Dillen, A. Polman, and A. van Blaaderen, Appl. Phys. Lett. 81, 838 (2002)

[77] T. van Dillen, A. Polman, C. M. van Kats, and A. van Blaaderen, Appl. Phys. Lett. 83, 4315 (2003)

[78] A. Mohraz and M. J. Solomon, Langmuir 21, 5298 (2005)

[79] C. J. Hernandez and T. G. Mason, J. Phys. Chem. C 111, 4477 (2007)

[80] J. Moon, A. Kim, J. Crocker, and S. Yang, Adv. Mater. 19, 2508 (2007)

[81] J. A. Champion, Y. K. Katare, and S. Mitragotri, Proc. Natl. Acad. Sci. U.S.A. 104, 11901 (2007)

[82] N. Yanai and S. Granick, Angew. Chem. 124, 5736 (2012)

[83] A. Tao, P. Sinsermsuksakul, and P. Yang, Nat. Nanotechnol. 2, 435 (2007).

[84] L. Zhang, G. Feng, Z. Zeravcic, T. Brugarolas, A. J. Liu, and D. Lee, ACS Nano 7, 8043 (2013).

[85] B. D. Fernandes, M. Spuch-Calvar, H. Baida, M. Tréguer-Delapierre, J. Oberlé, P. Langot, and J. Burgin, ACS Nano 7, 7630 (2013).

[86] T. K. Sau and A. L. Rogach, Adv. Mater. 22, 1781 (2010)

[87] Y. Xia, Y. Xiong, B. Lim, and S. Skrabalak, Angew. Chem., Int. Ed. 48, 60 (2009).

[88] A. Désert, C. Hubert, Z. Fu, L. Moulet, J. Majimel, P. Barboteau, A. Thill, M. Lansalot, E. Bourgeat-Lami, E. Duguet, and S. Ravaine, Angew. Chem., Int. Ed. 52, 11068 (2013)

[89] G. Odriozola, F. Jimenez-Angeles, and M. LozadaCassou, J. Chem. Phys. 129, 111101 (2008), arXiv:1010.3000 [cond-mat.mes-hall]

[90] P.-M. König, R. Roth, and S. Dietrich, Europhys. Lett. 84, 68006 (2008).

[91] G. Odriozola and M. Lozada-Cassou, Phys. Rev. Lett. 110, 105701 (2013)

[92] S. Sacanna and D. J. Pine, Curr. Opin. Colloid Interface Sci. 16, 96 (2011)

[93] C. R. Iacovella, R. E. Rogers, S. C. Glotzer, and M. J. Solomon, J. Chem. Phys. 133, 164903 (2010), arXiv:1005.2628 [cond-mat.soft]

[94] C. P. Royall, W. C. K. Poon, and E. R. Weeks, Soft Matter 9, 17 (2013), arXiv:1205.6665 [cond-mat.soft]

[95] K. L. Young, M. R. Jones, J. Zhang, R. J. Macfarlane, R. Esquivel-Sirvent, R. J. Nap, J. Wu, G. C. Schatz, B. Lee, and C. A. Mirkin, Proc. Natl. Acad. Sci. U.S.A. 109, $2240(2012)$ 
[96] X. Ye, C. Jun, M. Engel, J. A. Millan, W. Li, L. Qi, G. Xing, J. E. Collins, C. R. Kagan, J. Li, S. C. Glotzer, and C. B. Murray, Nat. Chem. 5, 466 (2013).

[97] Z. Tang, Z. Zhang, Y. Wang, S. C. Glotzer, and N. A.
Kotov, Science 314, 274 (2006)

[98] E. Gilbert, D. Johnson, and S. Keerthi, IEEE J. Robotics and Automation 4, 193 (1988) 